\title{
Dravet Syndrome and SCNIA gene mutations: a review
}

\begin{abstract}
Introduction: Dravet's Syndrome (DS) is epilepsy syndrome that strikes individuals in early childhood, also known as Severe Myoclonic Epilepsy of Infancy (SMEI). DS clinical presentation involves normal psychomotor development during the first month of life; premature convulsive conditions, beginning mainly during the first year of life; deceleration of psychomotor development later and other neurological deficits, similar to cerebellar ataxia, which ultimately results in a condition of severe disability and therefore individuals unable to live independently. The types of seizures are various and include clonic and tonic seizures, generalized or unilateral; myoclonic seizures and of absences.
\end{abstract}

Objective: Knowing that DS has as important etiology genetic disorders, this review aims to shed light on the main mutations that occur in the SCN1A gene, which considered the main gene related to SMEI and thus to correlate with its phenotype.

Methods: The databases PubMed, Scielo, Medscape and Google Scholar helped searching articles in both Portuguese and English.

Discussion: The SCN1A gene has 26 coding exons and translates the subunit 1 of the voltage-dependent neuronal sodium channel protein (Nav1.1). This protein consists of four domains (DI-IV), containing six transmembrane segments each. The subunit of the sodium channel is responsible for the formation of the transmembrane pore that allows the entry of sodium ions into the intracellular medium of the neurons. It is known that there are more than 500 distinct mutations at SCN1A gene associated with the DS.

Conclusion: Due to the impact of this syndrome on affected individuals and relatives lives, it is important to study the disease etiology. Therefore, this study is a review of several scientific publications encompassing mutations in the $S C N 1 A$ gene. In conclusion, the main kind of SCN1A gene mutations consist of missense and truncating mutations.

Keywords: dravet's syndrome, myoclonic epilepsy, SCN1A gene, subunit 1 of the voltage-dependent neuronal sodium channel protein (Nav1.1)
Volume 10 Issue 2 - 2020

\author{
Letícia Ferreira Marques da Silva, ${ }^{1,2}$ Gabriela \\ Siqueira Turolla,' Manoela Marques Ortega, ${ }^{2}$ \\ Paulo Henrique Pires de Aguiar ${ }^{2-6}$ \\ 'Medical Student, São Francisco University Medical School \\ (USF), Bragança Paulista, Brazil \\ ${ }^{2}$ Laboratory of Cell and Molecular Tumor Biology and Bioactive \\ Compounds, São Francisco University Medical School, Bragança \\ Paulista, Brazil \\ ${ }^{3}$ Department of Molecular Biology, ABC Medical School, Brazil \\ ${ }^{4}$ Post-Graduate Program, Department of Neurosurgery, State \\ Public Service Hospital (IAMSPE), Brazil \\ ${ }^{5}$ Department of Neurosurgery, Santa Paula Hospital, Brazil \\ ${ }^{6}$ Department of Neurology, Pontifical Catholic University of São \\ Paulo, Brazil
}

Correspondence: Prof. Dr. Paulo Henrique Pires de Aguiar, MD, Ph.D, Pontifical Catholic University of São Paulo, Sorocaba, Brazil, R. Joubert Wey, 290 - Vila Boa Vista, - SP, I8030-070, Tel +55 I I 981 283344, Email phpaneuroci@gmail.com

Received: February 29, 2020 | Published: March 17, 2020

\section{Introduction}

Dravet's Syndrome (DS) is a progressive encephalopathy described in 1978 by Charlotte Dravet, also known as Severe Myoclonic Epilepsy of Infancy (SMEI), an epilepsy syndrome of infantile onset. ${ }^{1}$ Common DS clinical features are normal neuropsychomotor development during the first month of life; early symptoms seizures presentation, starting during the first year of life; developmental psychomotor delay; and development of neurological deficits, such as lack of coordination similar to cerebellar ataxia, leading to the development of a severe disability that renders individuals unable to live independently. ${ }^{2}$

There are several types of seizures, including tonic-clonic, absence seizures complex atypical or partial, generalized or unilateral, myoclonic (ranging from a minute to hours, or even comatose) and may occur during wakefulness or during sleep. First, convulsion seizures are often associated with fever, but feverish seizures occur later in the course of the disease. The syndrome is notable for seizure frequency and its resistance to large variety of antiepileptic drugs. Photosensitivity is also a frequent symptom. ${ }^{2,3}$

The clinical characteristics and comorbidities of the syndrome in four patients with DS, including dysautonomia, nutrition issues, autism characteristics, and a high rate of sudden unexpected death in epilepsy (SUDEP) were previously described. ${ }^{4}$ In addition, four DS patients presented long Q-T intervals, suspecting of cardiorespiratory arrest associated with SUDEP, and SCN1A mutations. Similarly, five of 232 DS patients presented abnormalities or changes in the heart structure and SCN1A mutations. ${ }^{4}$ That gene is responsible for coding a voltage-dependent neuronal sodium channel. ${ }^{5}$

Estimated disease incidence is 1 in 22,000 births, which fits it into the group of rare diseases $(1 / 2,000)$. Patients and families with DS must face common problems of a rare disease including, lack of awareness and knowledge, lack and/or diagnostic delay, difficulties in accessing healthcare and medication, and loneliness. ${ }^{3,5}$ In addition, about $80 \%$ of affected patients harbor SCN1A gene mutations.

The ILAE genetics commission report from $2010^{6}$ stated that "posttest genetic counselling is crucial to help the patient understand the test result and begin to digest it in the context of life circumstances"; therefore, it is important review clinical and SCN1Arelated mutations in DS patients.

\section{Methods}

This review was compiled using articles, bibliographic reviews and thesis obtained from PubMed, Scielo, Medscape and Google 
Scholar databases in both language, Portuguese and English. A webpage for Non-Governmental Organization related to DS was also investigated. Thus, 21 publications involving clinical features, DS pathophysiology, SCN1A mutation frequencies, DS diagnosis and epidemiology were included in the present review. In addition, it was added a single publication about SCN1A inherited mutations. Most of the studies have discussed the SCN1A mutations while some studies also have characterized them.

\section{Results and discussion}

The SCN1A gene has 26 coding exons, responsible for encoding of the alpha subunit of the voltage-dependent neuronal sodium channel (Nav1.1 channel protein). In humans, there are 9 different subunits (Nav 1.1-Nav1.9) encoded by the SCN1A-SCN11A genes (Figure 1). ${ }^{7,8}$ Mutations in these genes are responsible for causing epilepsy, highlighting the SCN1A gene in the genesis of DS.7 The dependent sodium channel voltage opens in response to depolarization membrane, allowing the entry of ions in the in the neuron. ${ }^{9}$ This activity is responsible for generate the action potential and propagate the electrical impulses to the synaptic terminals, which results in synaptic transmission. The Nav1.1 channel protein has four homologous domains (DI-DIV), and each domain has 6 transmembrane segments. Its expression occurs predominantly in the GABA inhibitory

\section{SCNIA gene mutations}

According to Gonsales, ${ }^{8}$ SCN1A gene mutations screening has proved to be highly important for patients with DS, revealing 16 potentially gene deleterious changes totalizing 81 investigated patients (Table 1). ${ }^{8}$ The most common mutation type was the missense mutation, which promotes the replacement of waste amino acid in the protein chain. Considering only the number of mutations per protein segment, there is a clear predominance of changes in the pore-forming regions, between segments S5 and S6 and in the N- and C-terminal portions in addition to the segment responsible for detecting voltage (S4) in some domains such as I and IV. ${ }^{8,10}$ However, when considering interneurons, that is, those that secrete the GABA neurotransmitter. Therefore, it is believed that hyperexcitability observed in cases of loss of function of the sodium channel, as observed in the DS and in some of the genetic epilepsy with febrile seizures plus (GEFS+) cases, would be the result of a dysfunction in the circuit's neuronal inhibitors. $^{8}$

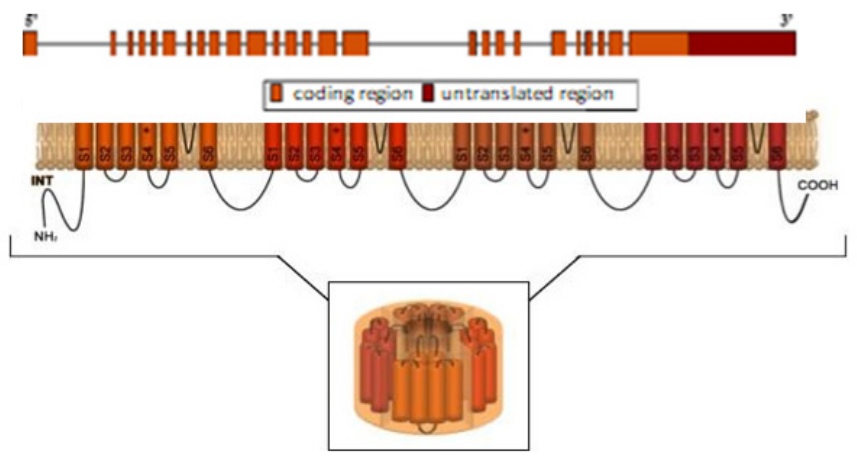

Figure I SCNIA gene structure and NavI.I channel protein. The SCNIA gene has 26 coding exons, responsible for encoding of the alpha subunit of the voltage-dependent neuronal sodium channel ( $\mathrm{Navl}$.I channel protein). The Navl.I channel protein has 4 homologous domains (DI-DIV) and each domain has 6 transmembrane segments. ${ }^{8}$

the amount of amino acid residues present in each region, most of the SCN1A mutations occurred at the conserved regions of the attenuated protein. Still, the S5-S6 region of the second domain stands out as presenting greater amount of mutations. Still, this approach highlights the frequency of changes in small segments, such as regions DIV/ S4-S5 (15 amino acid residues), DIII/S1-S2 (13 residues), DI/S3 (19 residues), DIV/S2- S3 (6 residues) and DI/S4-S5 (16 residues). Larger segments such as DI/S4-S5 (126 residues) and the N- and C-terminal regions (123 and 223 residues, respectively) shows a considerable reduction in SCN1A mutation frequency. To date (2017), about 774 mutations in SCN1A gene in DS patients has been described. ${ }^{8}$

Table I SCNIA gene mutation types, localization of the nucleotide changes, and alpha subunit of the voltage-dependent neuronal sodium channel protein phenotype in Dravet's syndrome

\begin{tabular}{|c|c|c|c|c|}
\hline Mutation type & Exon/Intron localization & Nucleotide changes & Protein phenotype & References \\
\hline Missense & Exon4 & c.530G>a & p.Gly I77Glu & (I) \\
\hline Missense & Exon5 & c. $680 \mathrm{~T}>\mathrm{G}$ & p.lle227Ser & ( 1 ) \\
\hline Missense & Exon26 & $c .5195 \mathrm{C}>\mathrm{T}$ & p.Proll 732Leu & (19) \\
\hline Missense & Exon26 & c. $5054 \mathrm{C}>\mathrm{T}$ & p.Ala $685 \mathrm{Val}$ & (13) \\
\hline Missense & Exon 15 & c. $2836 \mathrm{C}>\mathrm{T}$ & p.Arg946Cys & (19) \\
\hline Missense & Exon22 & $c .43 \mid 3 T>C$ & p.Met I438Thr & (19) \\
\hline Missense & Exon26 & c. $5555 \mathrm{~T}>\mathrm{C}$ & p.Met 1852Thr & (20) \\
\hline Missense & Exon26 & c. $4907 \mathrm{G}>\mathrm{A}$ & p.Arg1636Gln & (19) \\
\hline Missense & Exon6 & c.829T $>C$ & p.C277R & (8) \\
\hline Missense & Exon6 & c. $917 \mathrm{~A}>\mathrm{C}$ & p.H324P & (8) \\
\hline Missense & Exon 13 & c. $2360 \mathrm{~T}>\mathrm{G}$ & p.M787R & (8) \\
\hline
\end{tabular}


Table continue

\begin{tabular}{|c|c|c|c|c|}
\hline Mutation type & Exon/Intron localization & Nucleotide changes & Protein phenotype & References \\
\hline Missense & Exon2I & c. $4093 \mathrm{G}>\mathrm{T}$ & P.GI365C & $(8)$ \\
\hline Missense & Exon 16 & c. $2963 T>G$ & p.Leu998Arg & (II) \\
\hline Nonsense & Exon26 & c. $5674 \mathrm{C}>\mathrm{T}$ & p.Arg $1892 X$ & (13) \\
\hline Nonsense & Exon5 & c. $664 \mathrm{C}>\mathrm{T}$ & p.Arg222X & (I) \\
\hline Nonsense & Exon 12 & c. $2134 \mathrm{C}>\mathrm{T}$ & p.Arg7|2X & (13) \\
\hline Nonsense & Exon26 & c.5177G $>A$ & p.w1726X & (8) \\
\hline Splicing & Intron 2 & IVS2+IA>G & --- & (8) \\
\hline Splicing & Intron 4 & IVS4+IG>A & --- & (8) \\
\hline Splicing & Intron 8 & IVS8+3G>T & --- & (8) \\
\hline Splicing & Intron 2I & $|V S 2|+\mid G>A$ & --- & (8) \\
\hline Frameshift & Exon9 & c. I242delA & p. $1415 X$ & (8) \\
\hline Frameshift & Exon 19 & c.3719_3720insGATA & p.II 240fs XI244 & (8) \\
\hline Frameshift & Exon26 & c.5329delG & p.VI777fsXI778 & (8) \\
\hline In-frame deletions & Exon2 & c.296_313delTCTTCCGGTTCAGTGCCA & p.199_A 104del & (8) \\
\hline In-frame deletions & Exon 26 & c.5489_549IdelAGT & p.Q1830_FI83IdelinsL & (8) \\
\hline
\end{tabular}

As previously mentioned, regarding the mutations types among 24 publications, the most common was SCN1A missense mutation in exon $16 .{ }^{10}$ This variant is located within the DII S6 domain of the Scn1a protein and it was a novel de novo variant in the SCN1A gene. ${ }^{11}$ Another publication also has indicated missense (S123R, F1415I) and nonsense/truncating mutations (Y325X, R1407X, R1645X) in DS patients. ${ }^{2,7}$ Two other studies ${ }^{7,8}$ have described missense mutation (S1231R), and nonsense mutation (R1407X) whereas it mainly targets GABA inhibitory neurons while the electrophysiological properties of pyramidal neurons remain unchanged. Interestingly, the missense mutation (S1231R) has caused a single nucleotide alteration (c.3693T> A). ${ }^{7}$ Other common mutation type in DS is the frameshift mutation. ${ }^{8}$

Summarizing, missense mutation consists in one DNA nucleotide change in a way that the nucleotide triplet of which is part changes by encoding an incorrect amino acid. ${ }^{12}$ This can change the function of the protein in greater or lesser degree depending on the exon location and the importance of this particular amino acid. Nonsense/truncating mutation may change one of the nucleotides resulting in a termination codon in a way that the nascent protein is prematurely truncated. ${ }^{12,13}$ The frameshift mutation can be the result of insertion, duplication, and deletion nucleotides causing a reading grid landing. ${ }^{12}$

It worth to mention that DS less severe phenotype is more commonly associated with missense mutations than nonsense mutations. In addition, missense mutations in pore-forming region tend to result in more severe DS phenotype. ${ }^{14}$

\section{Genetic inheritance of mutations in SCN IA gene}

The SCN1A seizure disorder is normally autosomal dominant disease. ${ }^{15}$ A SCN1A seizure disorder patient may have an inherited or a de novo variant. The percentage of patients with an SCN1A seizure disorder with an affected relative decreases as the severity of the phenotype in the patient increases. Thus, most of the SCN1A-related SMEI result from a de novo variant. ${ }^{16}$ Each child with a $S C N 1 A$ seizure disorder has a $50 \%$ chance of inheriting the pathogenic variant; however, the risk of developing seizures is less than $100 \%$ because of reduced penetrance. Prenatal diagnosis for pregnancies at increased risk is possible if the pathogenic variant in the family is known..$^{16,17}$

On study has reported four DS patients harboring two SCN1A variants with possibly deleterious effect. One patient presented c.2816ARC/p.His939Pro and c.5364CRA/p.Asn1788Lys variants and other c.3235GRA/p.Val1079Ile variant, which was inherited from his asymptomatic father, and a de novo c.2504_2508delTTGAC mutation. The third patient carried the c.4723CRT/p.Arg1575Cys variant, inherited from his asymptomatic mother, and a de novo c. $1804 \mathrm{GRT} / \mathrm{p}$. Glu602X mutation. Finally, the fourth patient presented c.3325CRA/p. Pro1109Thr, inherited from his father, and a de novo c.4133delA mutation..$^{18}$ It was hypothesized that p.Val1079Ile, p.Pro1109Thr and p.Arg 1575Cys were novel rare non-synonymous polymorphisms, but it was not possible define which of the p.His939Pro and p.Asn1788Lys variants constituted the causative mutation, as the parents of the patient were unavailable for genetic analyses. Therefore, both were considered as potential missense mutations. ${ }^{19}$

Other study has evaluated 149 DS patients for SCN1A point mutations and both parents were available for genetic analyses. ${ }^{20}$ Direct sequencing failed to detect the mutation in either parent in 133 cases out of 149 (89\%), indicating that the mutation occurred de novo in those patients. For the 15 patients who inherited the mutation, it was inherited from the mother in five cases and from the father in ten. Two siblings (brother and sister) had the same mutation although it was undetectable in their parents. Interestingly, authors have found nine patients with novel recurrent mutations (i.e., an identical mutation 
in one patient) and 67 patients had mutations that have previously been reported by other authors. However, most mutations occurred de novo, indicating that mutations are repetitively generated through specific mechanisms. ${ }^{17}$

In conclusion, de novo mutations in $S C N 1 A$ gene occur in approximately $70-90 \%$ of all DS patients $(2,5,18)$. Inherited mutations are usually missense and occur often in a parent with a family history of febrile seizures or epilepsy with febrile seizures-plus (GEFS + ). ${ }^{5}$

Table 1 shows the main mutations observed in patients with syndrome DS. The literature shows around $80 \%$ of affected patients with DS carry a mutation in the SCN1A gene at $2 \mathrm{q} 24.3$, which encoding a voltage-gated sodium channel, and essential for the excitability of neurons. The majority of these variants are de novo mutations described as missense in exon 15 with the nucleotide change c. $2836 \mathrm{C}>\mathrm{T}$ and a protein phenotype p.Arg946Cys. In addition, it was described that mutations in exon 22 and 26, which present another SCN1A mutation types with different phenotypes due to the sodium channel alteration. In $5-10 \%$ of the cases, the variants are inherited (typically missense mutations), and the diagnosis is considered part of the febrile seizures or GEFS+ spectrum.

A minority of DS patients might have pathogenic variants in other genes such as PCDH19, SCN1B, SCN8A, HCN1, GABRA1, and $G A B R G 2$, and other pathologies close to DS should be considered for the differential diagnosis. ${ }^{19}$

Regarding the physiological and phenotypic consequences of the SCN1A mutations, it can be pointed out that exon 26 has a great importance in the genesis of DS, and the most cited in the literature. The SCN1A nonsense mutation at exon 26 results in the substitution of c. $5177 \mathrm{G}>\mathrm{A} / \mathrm{p} . \mathrm{W} 1726 \mathrm{X}$, generating an early termination codon, that corresponds to the pore formation region of the protein; thereby, impairing the inhibitory activity of the affected neurons. ${ }^{8}$ The nonsense mutation at exon 26, c. $5674 \mathrm{C}>\mathrm{T} / \mathrm{p}$. Arg $1892 \mathrm{X}$, leads to hemiclonic seizures at the age of five months; thereafter, the patient has presented other seizures including myoclonus daily from 1 year to 10 months of age, and generalized tonic clonic seizures starting at 1 year of age. The patient's IQ was 80 . The computed tomography and magnetic resonance imaging (MRI) of the patient were normal. The electroencephalogram analysis showed spike-wave complex and poly spike-wave complex. The magnetoencephalography had no cluster dipole. $^{13}$

The missense mutations at exon 26, c. $4907 \mathrm{G}>\mathrm{A} / \mathrm{p} . \mathrm{Arg} 1636 \mathrm{Gln}$, have been related to a phenotype of epileptic encephalopathy, myoclonic seizures, dystonia, and spasticity. In addition, when patients present the mutation c.5555T $>C$, also presented only afebrile generalized seizures. Few patients had myoclonic seizures and some had a clinical picture of SMEI. ${ }^{20}$

Regarding mutations in exon 4, c.530G $>$ A/p.Gly177Glu and exon 5 , c.680T $>$ G/p.Ile227Ser, DS patients have presented no history of acquired brain injury, normal cognitive and motor development, emergence of generalized or unilateral febrile or afebrile seizures in the first year of age, myoclonic spasms, intractable epilepsy, psychomotor delay after two years of onset seizures, normal MRI in the first year of seizure disorder, and a minimum follow-up of three years. ${ }^{1}$

Mutations IVS4 $+1 \mathrm{G}>\mathrm{A}$, IVS $8+3 \mathrm{G}>\mathrm{T}$ and IVS $21+1 \mathrm{G}>\mathrm{A}$ have revealed reduction in mutant allele splicing signal values. In addition, these mutations are located in the third nucleotide of the intron, which are less conserved than the first (9). However, the mutation IVS $2+1 \mathrm{~A}>\mathrm{G}$ involves a non-canonical sequence of the splicing donor site, i.e., not part of the most common mRNA splicing in eukaryotes. Mutations at splicing sites modify the normal signaling sequence and result in aberrant mRNA transcription, which may affect protein structure and function. However, to clarify this issue, functional or mRNA studies are required, which is difficult to obtain, since the SCN1A gene is only expressed in the central nervous system. ${ }^{15}$

Among the insertions and deletions, identified as c.1242delA, c.3719_3720insGATA and c.5329delG, cause the change of the amino acid residues of the protein sequence due to the promotion of premature termination of the chain by an appearance of an early stop codon. ${ }^{8}$ If the aberrant transcripts are translated, they would loss $3 / 4$ and half of the protein sequence for the c.1242delA deletion and for the c.3719 3720insGATA insertion, respectively. In addition, the protein sequence would loss the $\mathrm{C}$ terminal portion, which is crucial for protein anchoring on the membrane, for the c.5329delG deletion. ${ }^{8}$

Considering only the number of mutations per protein segment, there is a predominance of alterations in the pore formation regions, between segments S5 and S6, and in the N- and C-terminal portions, besides the segment responsible for voltage detection (S4) in some domains like I and IV.

\section{Conclusion}

Mutations in the SCN1A gene have a significant impact on the DS etiology. The overlapping mutations were missense and nonsense/ truncating mutations. Thus, the genetic test in SCN1A for DS clinical purposes is highly recommended.

\section{Acknowledgments}

None.

\section{Conflicts of interest}

The authors have no conflicts of interest to declare.

\section{Funding sources}

None.

\section{Author contributions}

LFMS and GST: carried out the bibliographic survey and wrote the paper. MMO: wrote the paper. PHPA: conception and design.

\section{References}

1. Nabbout R, Gennaro E, Dalla Bernardina B, et al. Spectrum of SCN1A mutations in severe myoclonic epilepsy of infancy. Neurology. 2003;60(12):1961-1967.

2. De Jonghe P. Molecular genetics of Dravet syndrome. Dev Med Child Neurol. 2011;53:7-10.

3. Dravet's Syndrome. Dravet Portugal. Lisboa, 2015.

4. Villas N, Meskis MA, Goodliffe S. Dravet syndrome: characteristics, comorbidities, and caregiver concerns. Epilepsy Behav. 2017;74:81-86.

5. Bayat A, Hjalgrim H, Møller RS. The incidence of SCN 1A-related Dravet syndrome in Denmark is 1: 22,000: A population-based study from 2004 to 2009. Epilepsia. 2015;56 (4):e36-e39. 
6. Scheffer IE, Berkovic S, Capovilla G, et al. Classificação da ILAE das epilepsias: artigo da posição da Comissão de Classificação e Terminologia da International League against Epilepsy. Epilepsia. 2012;53(Suppl 2):10-13.

7. Schutte SS, Schutte RJ, Barragan EV, et al. Model systems for studying cellular mechanisms of SCN1A-related epilepsy. $J$ Neurophysiol. 2016;115(4):1755-1766.

8. Gonsales MC. Estudos moleculares em epilepsias da infância e da adolescência: o potencial de aplicação clínica dos testes de genética molecular. Campinas. Tese [Fisiopatologia Médica] - Unicamp; 2013.

9. Brunklaus A, Zuberi SM. Dravet syndrome from epileptic encephalopathy to channelopathy. Epilepsia. 2014;55(7):979-984.

10. Fujiwara T. Clinical spectrum of mutations in SCN1A gene: severe myoclonic epilepsy in infancy and related epilepsies. Epilepsy Res. 2006;70:223-230

11. Pathirana B, Hettiarachchi D, Neththikumara NF, et al. A novel variant in SCN1A gene associated with Dravet syndrome. Seizure. 2019;69:213214.

12. Mutations type. Guía Metabólica. Barcelona, 2013.

13. Sugawara T, Mazaki-Miyazaki E, Ito M, et al. Nav1.1 mutations cause febrile seizures associated with afebrile partial seizures. Neurology. 2001;57(4):703-705.

14. Cetica V, Chiari S, Mei D, et al. Clinical and genetic factors predicting Dravet syndrome in infants with SCN1A mutations. Neurology. 2017;88(11):1037-1044.
15. Wang JW, Shi XY, Kurahashi H, et al. Epilepsy Genetic Study Group Japan. Prevalence of SCN1A mutations in children with suspected Dravet syndrome and intractable childhood epilepsy. Epilepsy Res. 2012;102(3):195-200.

16. Miller IO, Sotero de Menezes MA. SCN1A Seizure Disorders. In: Adam MP, Ardinger HH, Pagon RA, editors. GeneReviews ${ }^{\circledR}$ [Internet]. Seattle (WA): University of Washington, Seattle; 1993-2020.

17. Depienne C, Trouillard O, Saint-Martin C, et al. Spectrum of SCN1A gene mutations associated with Dravet syndrome: analysis of 333 patients. J Med Genet. 2009;46(3):183-191.

18. Steel D, Symonds JD, Zuberi SM, et al. Dravet syndrome and its mimics: Beyond SCN1A. Epilepsia. 2017;58(11):1807-1816.

19. Alame S, El-Houwayek E, Nava C, et al. Dravet Syndrome in Lebanon: First Report on Cases with SCN1A Mutations. Case Rep Med. 2019; 2019.

20. Mantegazza M, Gambardella A, Rusconi R, et al. Identification of an Nav1.1 sodium channel (SCN1A) loss-of-function mutation associated with familial simple febrile seizures. Proc Natl Acad Sci U S A. 2005;102(50):18177-18182.

21. Butler KM, da Silva C, Alexander JJ, et al. Diagnostic Yield From 339 Epilepsy Patients Screened on a Clinical Gene Panel. Pediatr Neurol. 2017;77:61-66. 